\title{
Isolation and Characterization of Methylammonium-resistant Mutants of Saccharomyces cerevisiae with Relieved Nitrogen Metabolite Repression of Allantoinase, Arginase and Ornithine Transaminase Synthesis
}

\author{
By W. J. MIDDELHOVEN \\ Laboratorium voor Microbiologie, Landbouwhogeschool, \\ Hesselink van Suchtelenweg 4, Wageningen, The Netherlands
}

(Received 21 September 1976; revised 3 February 1977)

\begin{abstract}
SUMMARY
The sensitivity of Saccharomyces cerevisiae $\alpha-\Sigma \mathrm{I} 278 \mathrm{~b}$ to methylammonium was dependent on the nature of the nitrogen source. Yeast strains carrying novel mutations, allNmr or argNmr, were isolated by selection for methylammonium resistance in media with allantoin or arginine as the sole nitrogen source. The mutations result in a considerable relief from nitrogen metabolite repression of allantoinase synthesis (allNmr), or of arginase and ornithine transaminase synthesis (argNmr and, to a lesser extent, allNmr). Both mutations were recessive to the wild type and were neither linked to each other, nor to the structural genes of allantoinase, arginase and ornithine transaminase. Hence they represent regulatory genes without operator characteristics. Experiments with ammoniumlimited continuous cultures indicated that ammonium transport was not affected by the allNmr and arg Nmr mutations. Ammonium repression of catabolic NADdependent glutamate dehydrogenase was also unaffected. The $\operatorname{argNmr}$ locus, in cooperation with other loci, appears to control nitrogen metabolite repression of the arginine degradative pathway; it is probably pathway-specific. The allNmr locus may have a general function in nitrogen metabolite repression of various degradative pathways since the allNmr mutation causes resistance to methylammonium in the presence of several metabolically unrelated nitrogen sources.
\end{abstract}

\section{INTRODUCTION}

Mutants of Aspergillus nidulans with impaired repression by ammonium of many enzymes involved in the degradation of nitrogenous substances have been isolated by selection for resistance to methylammonium (Arst \& Cove, I969). Methylammonium is considered to be a structural analogue of ammonium. In mutants resistant to methylammonium, designated meaR, the repression of numerous enzymes by ammonium is relieved. Although a common nitrogen metabolite repressor for all of these enzymes was assumed, in a subsequent paper (Arst \& Page, I973) methylammonium resistance was attributed to impairment of the common permease for ammonium and methylammonium. Likewise, a recent paper on methylammonium transport in a strain of Saccharomyces cerevisiae (Roon et al., $1975 a$ ) reveals that methylammonium resistance is associated with defective transport of and growth on ammonium. In the present study it is demonstrated that selection for methylammonium resistance is a valuable tool in the isolation of yeast mutants with impaired nitrogen metabolite repression of enzymes involved in the degradation of allantoin and arginine.

Saccharomyces cerevisiae is able to utilize exogenous allantoin as the sole source of 
nitrogen but is unable to produce allantoin by purine base catabolism (DiCarlo, Schultz \& McManus, I95I; LaRue \& Spencer, 1968). Allantoin is degraded in yeast to urea and glyoxylate by three successive hydrolytic enzyme reactions (DiCarlo, Schultz \& Kent, I953; Cooper \& Lawther, I973), the first being catalysed by allantoinase (allantoin amidohydrolase, EC 3.5.2.5). Allophanate, an intermediate of the urea degradative pathway in yeast (Roon \& Levenberg, 1970; Whitney \& Cooper, 1972), is considered to be the inducer of the whole pathway catalysing the conversion of allantoin into glyoxylate, ammonium and bicarbonate, since all of the enzymes appear to be uninducible in mutants unable to synthesize the compound (Cooper \& Lawther, 1973; Whitney, Cooper \& Magasanik, I973).

Allantoinase synthesis in protoplasts of $S$. carlsbergensis is repressed by nitrogenous compounds like glutamine, asparagine and ammonium (van de Poll, 1970). Bossinger, Lawther \& Cooper (1974) observed only a slight repression by ammonium but strong repression by glutamine, asparagine or serine when added singly, or by ammonium and alanine when added together, to a $S$. cerevisiae culture in glucose medium provided with a gratuitous inducer.

The pathway of arginine metabolism in $S$. cerevisiae is initiated by the successive actions of arginase (L-arginine amidinohydrolase, EC 3.5.3. I and ornithine transaminase (Lornithine:2-oxoacid aminotransferase, EC 2.6.I.13) (Middelhoven, I964). Yeast arginase is activated in vivo by ferrous ions; in vitro these can be replaced by other divalent cations. The enzyme is strongly competitively inhibited by ornithine (Middelhoven, 1969a; Middelhoven, de Waard \& Mulder, 1969). The syntheses of arginase and ornithine transaminase are regulated by induction and repression. Arginine and ornithine serve as the inducers (Middelhoven, 1964, I970). Fully induced enzyme activities are observed after growth on arginine as the sole nitrogen source. The addition of readily assimilable nitrogenous compounds like ammonium, asparagine, aspartate, glutamine or alanine results in much lower enzyme activities; however these are higher than those produced in the absence of inducer (Middelhoven, 1970). Nitrogen metabolite repression is indicated, in particular, by the strong derepression of both enzymes during nitrogen starvation in the absence of inducers (Middelhoven, 1969, 1970; Whitney \& Magasanik, 1973). Myo-inositol probably plays a role in this process (Middelhoven, $1969 b$ ). No evidence was obtained for carbon catabolite repression or for inactivation repression (Middelhoven, $1969 c, 1970$ ).

Yeast mutants $(g d h A)$ lacking anabolic NADP-specific glutamate dehydrogenase [Lglutamate:NADP ${ }^{+}$oxidoreductase (deaminating), EC I .4.I.4] and characterized by their slow growth in ammonium medium, were claimed to have lost nitrogen metabolite repression of a general amino-acid permease (Grenson \& Hou, 1972), allantoinase and arginase but not of ornithine transaminase or catabolic NAD-specific glutamate dehydrogenase [L-glutamate: $\mathrm{NAD}^{+}$oxidoreductase (deaminating), EC I.4.I.2] (Dubois, Grenson \& Wiame, 1973, 1974). Nitrogen metabolite repression was considered to result from the interference of the NADP-specific glutamate dehydrogenase molecule with the process of enzyme synthesis (Dubois et al., 1974). The role of $g d h A^{+}$in nitrogen metabolite repression in yeast has been questioned by several authors (van de Poll, 1973; Bossinger \& Cooper, 1975; Roon, Larimore \& Levy, 1975b).

The mutation $g d h C R$ has also been claimed to result in the mitigation of nitrogen metabolite repression of arginase and allantoinase (Dubois \& Grenson, 1974). In gdhCR mutants the synthesis of catabolic NAD-specific glutamate dehydrogenase is also derepressed.

As in yeast, $g d h A$ mutants of $A$. nidulans exhibit impaired ammonium repression of several enzymes (Kinghorn \& Pateman, 1973; Arst \& MacDonald, 1973), although the 
Table I. Yeast strains used

All strains are derived from the isonuclear wild-type strains $\alpha-\Sigma 1278 b$ and a-3962c.

Strain

\begin{tabular}{|c|c|}
\hline$\alpha$-HPIOO & wild type \\
\hline a-LAIOO & wild type \\
\hline D4OE & wild type \\
\hline $\mathrm{D} g d h A I$ & $g d h A$ \\
\hline$\alpha$-AllNmr-5 & allNmr \\
\hline D252E & allNmr/wild type \\
\hline D246E & allNmr \\
\hline a-ArgNmr-12 & $\operatorname{argNmr}$ \\
\hline $25 I E$ & $\operatorname{argNmr} /$ wild type \\
\hline $2245 E$ & $\operatorname{argNmr}$ \\
\hline
\end{tabular}

Genotype

$$
\begin{aligned}
& \alpha \text {-his, ura } \\
& \text { a-leu, ade } \\
& \alpha-h i s, \text { ura, } \text { eth }^{\mathrm{R}} / \mathrm{a}-l e u, \text { ade } \\
& \alpha \text {-ile, gdhAI/a-ade, gdhAI, eth }{ }^{\mathrm{R}} \\
& \alpha \text {-his, ura, allNmr } 5 \\
& \alpha \text {-leu, his, ura, eth } h^{\mathrm{R}} \text {, allNmr5/a-ade } \\
& \alpha \text {-leu, his, ura, eth }{ }^{\mathrm{R}}, \text { allNmr5/a-ade, eth }{ }^{\mathrm{R}} \text {, allNmr5 } \\
& \text { a-leu, ade, argNmri } 2 \\
& \alpha \text {-his, } \text { eth }^{\mathrm{h}}, \operatorname{argNmri2/\mathrm {a}-leu,\text {ade}}
\end{aligned}
$$

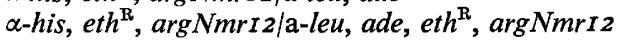

repression by glutamine and glutamate is retained (Hynes, 1974). Enzymes involved in arginine or allantoin catabolism were not included in these studies.

In the present study, the repression of allantoinase, arginase and ornithine transaminase by several nitrogenous compounds is investigated in the wild type, in a $g d h A$ mutant and in two methylammonium-resistant mutants (all $\mathrm{Nmr}$ and $\operatorname{argNmr}$ ).

\section{METHODS}

Yeast strains and genetic techniques. All strains used (Table I) were derived from two isonuclear wild-type strains of $S$. cerevisiae, $\alpha-\Sigma \mathrm{I} 278 \mathrm{~b}$ and a-3962c, kindly provided by Dr J. M. Wiame, Brussels. The origin of the strains has been described previously (Béchet, Grenson \& Wiame, 1970). Auxotrophic mutants of these strains were selected by the method of Horn \& Wilkie (I966). Mutants lacking NADP-specific glutamate dehydrogenase $(g d h A)$, arginase, ornithine transaminase or allantoinase were isolated on phloxine $\mathrm{B}$ medium (Middelhoven, Broekhuizen \& van Eijk, I976b). Ethyl methanesulphonate (I \%, $\mathrm{v} / \mathrm{v}$ ) was used for mutagenesis; excess reagent was removed from the cells by suspending them in $10 \%(\mathrm{w} / \mathrm{v})$ sodium thiosulphate and subsequent washing (Parker \& Mattoon, I969). Mating of strains and sporulation of diploids were achieved by conventional techniques (Fink, 1970), except that glucose was replaced by maltose in the pre-sporulation medium. Ascospores were separated by ultrasonic treatment. Auxotrophic haploid segregants were recognized as described above (Horn \& Wilkie, 1966) and were screened for their nutritional requirements by replica plating. Respiratory-deficient strains were identified by their inability to grow on yeast extract/ethanol/peptone agar and were discarded. The mating type of the segregants was deduced from their ability to complement other auxotrophic strains of different nutritional requirement and known mating type. Following this procedure, two auxotrophic segregants were obtained, $\alpha$-HPIOO $(\alpha$-his, ura) and a-LAIOO (a-leu, ade), which were used as parent strains in the isolation of methylammonium-resistant mutants. To obtain these mutants, cells of both strains were treated with ethyl methanesulphonate and spread on glucose/methylammonium agar with allantoin or arginine as the nitrogen sources. The concentration of methylammonium chloride was $50 \mathrm{~mm}$ in glucose/allantoin agar and $500 \mathrm{~mm}$ in glucose/arginine agar; L-histidine, uracil, L-leucine and adenine were added at $200 \mathrm{mg} \mathrm{l}^{-1}$ if required. Resistant mutants produced colonies in about 3 to 7 days and were streaked on medium of the same composition. Pure cultures were maintained on yeast extract/glucose/peptone agar.

Prototrophic diploid strains, homozygous with respect to the methylammonium 
resistance marker, were obtained by mating a methylammonium-resistant mutant with a methylammonium-sensitive strain of complementary nutritional requirement, which had previously been mutated to resistance to $50 \mathrm{mg}$ DL-ethionine $1^{-1}$. This resistance is recessive to wild type and is presumably due to the lack of specific methionine permease (Gits \& Grenson, 1967) rather than of a general amino-acid permease since the wild-type sensitivity to Io $\mathrm{mg}$ L-canavanine sulphate $1^{-1}$ had been retained. After sporulation of the diploid and ultrasonic separation of the ascospores, the suspension was plated on glucose/ammonium agar supplemented with DL-ethionine $\left(25 \mathrm{mg} \mathrm{l}^{-1}\right)$ and the nitrogenous substances required by the parent strains (each at $200 \mathrm{mg} \mathrm{l}^{-1}$ ). Segregant strains were screened for methylammonium resistance (see below), nutritional requirements, respiratory ability and mating type. Diploids were constructed in the usual way from methylammonium-resistant segregants of opposite mating type and complementary nutritional requirements.

Growth conditions. The growth medium contained $40 \mathrm{~g}$ glucose $\mathrm{1}^{\mathbf{1}}$; mineral constituents, vitamins and buffer were added as described previously (Middelhoven, 1969c). Nitrogen sources were each added to a concentration of 40 mM-nitrogen. The medium, $\mathrm{pH} 5.5$, was sterilized for $\mathrm{I} 5 \mathrm{~min}$ at $\mathrm{I} 20^{\circ} \mathrm{C}$; glucose was autoclaved separately and labile nitrogen sources were sterilized by Seitz filtration. Solid media contained $20 \mathrm{~g}$ agar $1^{-1}$.

Portions of medium (10 to $20 \mathrm{ml}$ ) in $100 \mathrm{ml}$ conical flasks were inoculated with $0.1 \mathrm{ml}$ of a pre-culture in the same medium and incubated in a rotary shaker at $30^{\circ} \mathrm{C}$ until no more than half the maximum cell yield (on the basis of $40 \mathrm{~mm}$-nitrogen) was obtained; this usually took about $\mathrm{I} 6 \mathrm{~h}$. Growth was monitored turbidimetrically. For some experiments larger amounts of yeast cells were grown in Kluyver flasks.

The substrate constant for ammonium and the maximum specific growth rate in ammonium-limited chemostat cultures were determined as described by Middelhoven et al. (1976a).

Resistance to methylammonium was observed in liquid media containing different nitrogen sources and variable concentrations of methylammonium chloride. Portions of medium $(2 \mathrm{ml})$ in test tubes were inoculated with $\mathrm{I} \%(\mathrm{v} / \mathrm{v})$ of a fully grown pre-culture in Io mMglutamate medium and observed visually at intervals over 3 days. Replica plating was not suitable for demonstrating methylammonium resistance.

Enzyme determinations. Preparation of cell-free extracts, protein determination, activation of arginase with $\mathrm{MnCl}_{2}$ and determination of ornithine transaminase were as described by Middelhoven (I964). However, in the present study, the glycinate buffer was omitted from the incubation mixtures for the arginase assay because $100 \mathrm{mM}-\mathrm{L}$-arginine $\mathrm{pH} 9.5$ buffered the system sufficiently by itself, and urea was determined with $p$-dimethylaminobenzaldehyde by the method of Hagan \& Dallam (I968). This latter method was found to be satisfactory provided that arginine was added to the urea standard series in amounts equal to those present in the samples. Incubation mixtures for the allantoinase assay contained (in a total volume of $\mathrm{I} \cdot 0 \mathrm{ml}): 0.2 \mathrm{ml}$ cell-free extract $(0.5$ to $2 \mathrm{mg}$ protein), 10 $\mu \mathrm{mol}$ $\mathrm{Na}_{2}$ EDTA pH $7 \cdot 2$, $100 \mu \mathrm{mol} \mathrm{Tris} / \mathrm{HCl}$ buffer $\mathrm{pH} 7.2$ and $30 \mu \mathrm{mol}$ allantoin, added in that order. After $\mathrm{I} \mathrm{h}$ at $30^{\circ} \mathrm{C}$, the reaction was stopped by the addition of $\mathrm{I} \cdot 0 \mathrm{ml} \mathrm{I} \mathrm{M}$-perchloric acid and $8.0 \mathrm{ml}$ water. Samples of the supernatant fluid were heated for $10 \mathrm{~min}$ at $100{ }^{\circ} \mathrm{C}$ to convert the reaction product, allantoic acid, to glyoxylic acid which was then determined by the method of Vogels \& van der Drift (I970). Conditions were such that no more than $2.5 \mu \mathrm{mol}$ allantoin was hydrolysed during the enzyme reaction. Glutamate dehydrogenases were determined according to Doherty (1970). All specific enzyme activities were expressed as $\mu \mathrm{mol}$ substrate converted $\mathrm{h}^{-1}(\mathrm{mg} \text { protein })^{-1}$ at $30^{\circ} \mathrm{C}$. 


\section{RESULTS}

Methylammonium chloride inhibits growth of $S$. cerevisiae strain $\alpha-\Sigma_{1} 278 b$ when added to growth media containing arginine, allantoin or certain other nitrogenous compounds as the sole nitrogen source. Mutants resistant to methylammonium chloride were selected in two auxotrophic strains, $\alpha$-HPIOO and a-LAIOO, by spreading cells treated with ethyl methanesulphonate on agar media containing the analogue and different nitrogen sources. Two hundred of the mutants obtained were screened for metabolite repression of the enzymes allantoinase, arginase and ornithine transaminase.

Dominance or recessivity of the resistance to methylammonium was recognized from the properties of heterozygous diploids obtained by mating mutants to wild-type strains. Complementation of the different mutations was studied in diploids obtained by mating methylammonium-resistant mutants of $\alpha$-HPIOO with those of a-LAIOO. On the basis of these preliminary experiments, methylammonium-resistant yeast strains showing impaired nitrogen metabolite repression in allantoin or arginine catabolism were divided into two groups, designated allNmr and argNmr. Mutants belonging to the former group, isolated on the basis of methylammonium resistance in the presence of various nitrogen sources (DL-allantoin, urea, DL-2-aminobutyrate, L-arginine), were recessive to wild type and showed impaired nitrogen metabolite repression with regard to allantoinase synthesis and, to a lesser extent, with regard to arginine catabolism. $\operatorname{argNmr}$ mutants, exclusively isolated on methylammonium/arginine medium, were also recessive to wild type but showed impaired nitrogen metabolite repression only in arginine catabolism, allantoinase synthesis being controlled as in the wild-type strains. Mutants representative of the two groups, $\alpha$-AllNmr-5 and a-ArgNmr-I2, were crossed with ethionine-resistant wild-type strains. The resulting diploids were sporulated and segregant strains were isolated on the basis of ethionine resistance. Amongst these segregants there was a $I: I$ distribution of wild and Nmr phenotypes, indicating that the mutations were of a nuclear type and not linked to the ethionine resistance marker. Moreover, a complete correlation was observed between methylammonium resistance and impaired nitrogen metabolite repression. For detailed physiological studies, prototrophic diploid strains, homozygous for the $\mathrm{Nmr}$ markers, were preferable to haploid auxotrophic strains, as the latter generally show lower maximum specific growth rates. In this way, any effect of growth rate or auxotrophy on nitrogen metabolite repression is precluded as far as possible.

\section{Methylammonium resistance}

A wild-type diploid yeast strain (D4OE), diploid yeast strains homozygous with respect to the $g d h A$, allNmr and $\operatorname{argNmr}$ mutations, and heterozygotes allNmr/wild type and $\operatorname{argNmr} /$ wild type were grown at various methylammonium concentrations $(0, \mathrm{I}, 2,5$, Io, 20, 50, 100, 200, 500, 750 and $1000 \mathrm{mM}$ ) in media with different nitrogen sources. The threshold concentrations of methylammonium toxicity, shown in Table 2, represent the lowest concentrations at which a.decreased growth rate was observed. The sensitivity of the wild-type strain D4OE to methylammonium varied greatly with the nitrogen source. In the presence of allantoin, growth was inhibited at $2 \mathrm{mM}$, whereas in the presence of ammonium or of nitrogen sources from which ammonium is readily generated (e.g. asparagine, glutamine) concentrations of $750 \mathrm{~mm}$ were tolerated. The methylammonium sensitivity of $\operatorname{Dg} d h A I$ was about the same as that of the wild type except for slightly increased resistance in urea, allantoin and proline media and increased sensitivity in leucine and serine media. The arg Nmr diploid D245E showed marked resistance to methylammonium 


\section{Table 2. Threshold concentrations of methylammonium toxicity}

The concentrations (mM) listed are the lowest at which inhibition of the growth rate was observed.

\section{Yeast stra
Genotyp}

Nitrogen source
Ammonium

DL-Alanine

DL-Allantoin

DL-2-Aminobutyrate

4-Aminobutyrate

L-Arginine

L-Asparagine

L-Aspartate

L-Citrulline

L-Glutamate

L-Glutamine

L-Isoleucine

L-Leucine

L-Ornithine

L-Proline

L-Serine

L-Threonine

L-Valine

Urea

$\begin{array}{rr}>1000 & 1000 \\ 750 & 1000 \\ 2 & 5 \\ 50 & 100 \\ 50 & 50 \\ 200 & 200 \\ 1000 & 1000 \\ 500 & 500 \\ 100 & 100 \\ 50 & 50 \\ 1000 & >1000 \\ 50 & 100 \\ 500 & 50 \\ 50 & 50 \\ 50 & 100 \\ 500 & 100 \\ 50 & 100 \\ 200 & 100 \\ 50 & 200\end{array}$

\begin{abstract}
D245E
$\operatorname{argNmr}$
\end{abstract}

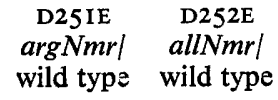

wild type wild type

in arginine medium and increased resistance in the presence of 2-aminobutyrate, glutamate and leucine. The allNmr diploid D246E showed increased resistance to methylammonium in the presence of a number of nitrogen sources (allantoin, 2-aminobutyrate, arginine, glutamate, isoleucine, ornithine, proline, threonine, valine and urea). Since many of these compounds are unrelated structurally and metabolically, the allNmr locus probably has a general function not restricted to allantoin catabolism. The heterozygous diploids D25IE and D252E behaved in essentially the same way as the wild type. The allNmr and argNmr mutations are clearly recessive to wild type.

\section{Allantoinase synthesis}

The effects of the allNmr, argNmr and $g d h A$ mutations on allantoinase synthesis are shown in Table 3. In the wild-type diploid D4OE, allantoinase was produced at a basal level after growth in the presence of readily assimilable nitrogen sources such as ammonium, glutamine, asparagine, ammonium/glutamate and ammonium/aspartate. The same nitrogen sources efficiently repressed allantoinase synthesis when added together with allantoin, arginine or urea. Growth of the wild-type strain $\mathrm{D} 4 \mathrm{OE}$ in the presence of all other nitrogen sources tested resulted in higher specific allantoinase activities. It is not clear whether allantoinase synthesis requires the presence of the urea catabolite allophanate as an internal inducer, as was observed in another yeast strain (Cooper \& Lawther, 1973). Specific allantoinase activities after growth on allophanate-generating nitrogen sources like allantoin, urea and arginine were lower than those after growth on DL-alanine, DL-2-aminobutyrate and other amino acids (e.g. valine, leucine and methionine; not shown). However, an essential role of allophanate in allantoinase synthesis cannot be ruled out on the basis of this experiment.

The diploid strain DgdhAI, lacking NADP-specific glutamate dehydrogenase, showed a 
Table 3. Specific allantoinase activities in diploid yeast strains grown in glucose media with different nitrogen sources

The results are expressed as $\mu \mathrm{mol}$ allantoin hydrolysed $\mathrm{h}^{-1}$ (mg protein) ${ }^{-1}$ under standard conditions and indicate the extremes of at least four determinations.

$\begin{array}{llccccc}\text { Yeast strain } & \ldots & \text { D4OE } & \mathrm{D} g d h A I & \mathrm{D} 246 \mathrm{E} & \mathrm{D} 252 \mathrm{E} & \mathrm{D} 245 \mathrm{E} \\ \text { Genotype } & \ldots & \text { wild type } & g d h A & \text { allNmr } & \begin{array}{c}\text { allNmrl } \\ \text { wild type }\end{array} & \text { argNmr }\end{array}$

Nitrogen source

\begin{tabular}{|c|c|c|c|c|c|}
\hline $\mathrm{NH}_{4}{ }^{+}$ & $0.2-0.6$ & $I \cdot 4-1 \cdot 6^{*}$ & $0.9-I \cdot 0$ & $0.3-0.4$ & $0.4-0.5$ \\
\hline L-Glutamate & $1 \cdot 0-2 \cdot 2$ & $I \cdot I-2 \cdot I$ & $I \cdot 2-I \cdot 3$ & $I \cdot 2-1 \cdot 5$ & $1 \cdot 0-1 \cdot 5$ \\
\hline L-Glutamine & $0.2-0.3$ & $0.3-0.6$ & $0 \cdot 9-I \cdot 2$ & - & - \\
\hline L-Asparagine & $0.3-0.4$ & $0.3-0.7$ & $0.8-0.9$ & $0.1-0.2$ & - \\
\hline $\mathrm{NH}_{4}{ }^{+}+\mathrm{L}$-Glutamate & $0.1-0.2$ & $0 \cdot 6-1 \cdot 2$ & $I \cdot I-I \cdot 3$ & $0.2-0.3$ & - \\
\hline $\mathrm{NH}_{4}++$ L-Aspartate & $0.1-0.2$ & $0.2-0.3$ & $0 \cdot 7-I \cdot 3$ & - & - \\
\hline $\begin{array}{l}\text { DL-Alanine } \\
\quad+\mathrm{NH}_{4}{ }^{+}\end{array}$ & $\begin{array}{l}4 \cdot 5-6 \cdot 8 \\
0 \cdot 5-1 \cdot 0\end{array}$ & $\begin{array}{l}5 \cdot 2-7 \cdot 8 \\
2 \cdot 3-2 \cdot 9\end{array}$ & $\begin{array}{l}5 \cdot I-8 \cdot 5 \\
3 \cdot 5-8 \cdot 9\end{array}$ & - & - \\
\hline $\begin{array}{l}\text { DL-2-Aminobutyrate } \\
\quad+\mathrm{NH}_{\mathbf{4}}{ }^{+} \\
\quad+\mathrm{L}-\text { Asparagine }\end{array}$ & $\begin{array}{l}2 \cdot 2-9 \cdot 2^{*} \\
0 \cdot 6-1 \cdot 0 \\
-\end{array}$ & $\begin{array}{l}3 \cdot I-5 \cdot 4^{*} \\
2 \cdot 3-4 \cdot 5 \\
I \cdot 4-2 \cdot I\end{array}$ & $\begin{array}{l}5 \cdot 4-6 \cdot 7^{*} \\
4 \cdot 8-5 \cdot 3 \\
3 \cdot 7-5 \cdot 4\end{array}$ & - & E \\
\hline $\begin{array}{l}\text { DL-Allantoin } \\
\quad+\mathrm{NH}_{4}^{+} \\
\quad+\mathrm{L} \text {-Glutamate } \\
\text { + L-Glutamine } \\
\text { + L-Asparagine } \\
\quad+\mathrm{NH}_{4}{ }^{+}+\mathrm{L} \text {-Glutamate }\end{array}$ & $\begin{array}{l}2 \cdot 5-4 \cdot 0 \\
0 \cdot 4-0 \cdot 6 \\
I \cdot 8-I \cdot 9 \\
0 \cdot 3-0 \cdot 4 \\
0 \cdot 3-0 \cdot 4 \\
0 \cdot I-0 \cdot 4\end{array}$ & $\begin{array}{l}2 \cdot 9-4 \cdot 7^{*} \\
2 \cdot 8-3 \cdot 0 * \\
1 \cdot 1-4 \cdot 2 \\
0 \cdot 3-0 \cdot 7 \\
0 \cdot 3-0 \cdot 7 \\
0 \cdot 6-1 \cdot 6\end{array}$ & $\begin{array}{l}I \cdot 9-2 \cdot 6 \\
I \cdot 5-I \cdot 9 \\
I \cdot 4-2 \cdot 0 \\
I \cdot 5-I \cdot 6 \\
0 \cdot 9-I \cdot 1 \\
I \cdot 4-I \cdot 5\end{array}$ & $\begin{array}{l}2 \cdot 7-3 \cdot 1 \\
0.5-0 \cdot 8 \\
1 \cdot 5-1 \cdot 9 \\
- \\
0 \cdot 1-0 \cdot 2 \\
0 \cdot 2-0.3\end{array}$ & $\begin{array}{l}3 \cdot 0-3 \cdot 2 \\
0 \cdot 4-0 \cdot 7 \\
1 \cdot 0-2 \cdot 2 \\
- \\
0 \cdot 1-0 \cdot 2 \\
0 \cdot 1-0 \cdot 2\end{array}$ \\
\hline $\begin{array}{l}\text { L-Arginine } \\
\quad+\mathrm{NH}_{4}^{+} \\
\quad+\mathrm{L}-\mathrm{Glutamine} \\
\quad+\mathrm{L}-\text { Asparagine } \\
\quad+\mathrm{NH}_{4}{ }^{+}+\mathrm{L} \text {-Glutamate }\end{array}$ & $\begin{array}{l}2 \cdot 2-3 \cdot 2 \\
0 \cdot 6-I \cdot 2 \\
0 \cdot 3-0 \cdot 4 \\
0 \cdot I-0 \cdot 2 \\
0 \cdot 2-0 \cdot 3\end{array}$ & $\begin{array}{l}1 \cdot 8-3 \cdot 6 \\
I \cdot 9-3 \cdot 2 \\
0 \cdot 2-0 \cdot 3 \\
0 \cdot 3-0 \cdot 4 \\
0 \cdot 5-0 \cdot 8\end{array}$ & $\begin{array}{l}1 \cdot 6-4 \cdot 4 \\
\mathrm{I} \cdot 6-4 \cdot 5 \\
\mathrm{I} \cdot 2-\mathrm{I} \cdot 8 \\
0 \cdot 5-0 \cdot 7 \\
1 \cdot 6-3 \cdot 3\end{array}$ & $\begin{array}{l}2 \cdot 6-2 \cdot 8 \\
0.6-0.9 \\
- \\
0.1-0.2 \\
0.1-0.2\end{array}$ & $\begin{array}{l}0 \cdot 6-1 \cdot 3 \\
0 \cdot 7-0.9 \\
-1-0.2 \\
0 \cdot 1-0 \cdot 2 \\
0 \cdot 3-0.4\end{array}$ \\
\hline $\begin{array}{l}\text { Urea } \\
\quad+\mathrm{NH}_{4}{ }^{+} \\
\quad+\mathrm{L}-\mathrm{Glutamine} \\
\quad+\mathrm{L}-\text { Asparagine } \\
\quad+\mathrm{NH}_{4}{ }^{+}+\mathrm{L}-\text { Glutamate }\end{array}$ & $\begin{array}{l}2 \cdot 7-3 \cdot 4 \\
I \cdot 3-3 \cdot I \\
0 \cdot 8-I \cdot I \\
0 \cdot 3-0 \cdot 5 \\
0 \cdot 2-0 \cdot 3\end{array}$ & $\begin{array}{l}2 \cdot 4-2 \cdot 7^{*} \\
3 \cdot I-3 \cdot 5^{*} \\
I \cdot 9-2 \cdot 2 \\
0 \cdot 4-I \cdot 0 \\
0 \cdot 8-2 \cdot 4\end{array}$ & $\begin{array}{l}\mathrm{I} \cdot 2-2 \cdot 5 \\
\mathrm{I} \cdot 4-2 \cdot 0 \\
2 \cdot 7-2 \cdot 9 \\
0 \cdot 8-\mathrm{I} \cdot 2 \\
2 \cdot 1-2 \cdot 4\end{array}$ & $\begin{array}{l}2 \cdot 3-2 \cdot 4 \\
I \cdot I-I \cdot 6 \\
- \\
0 \cdot 2-0 \cdot 3 \\
0 \cdot 2-0 \cdot 3\end{array}$ & $\begin{array}{l}\mathrm{I} \cdot 9-2 \cdot 8 \\
\mathrm{I} \cdot 3-\mathrm{I} \cdot 7 \\
= \\
0 . \mathrm{I}-0 \cdot 3\end{array}$ \\
\hline
\end{tabular}

* Specific growth rates, about $0.15 \mathrm{~h}^{-1}$; specific growth rates of other cultures, 0.35 to $0.4 \mathrm{I} \mathrm{h}^{-1}$.

different regulation of allantoinase synthesis. Repression by ammonium, which is slowly assimilated by this strain, was weak but repression by glutamine and asparagine was almost as strong as in the wild-type strain D4OE (cf. van de Poll, 1973). However, in the allNmr diploid D246E, allantoinase synthesis during growth in the presence of glutamine, asparagine, ammonium/glutamate and ammonium/aspartate was considerable. Obviously the nitrogen metabolite repression of allantoinase synthesis was greatly relieved by the allNmr mutation. This was not the case with the argNmr mutation (D245E) which had no effect on allantoinase synthesis. It is concluded that the allNmr mutation is recessive to wild type since the heterozygous diploid D252E behaves like the wild-type diploid D4OE.

\section{Arginine catabolism}

Average specific activities of arginase and ornithine transaminase in diploid strains grown with different nitrogen sources are given in Table 4 . In all strains the presence of arginine or another inducer in the medium was required for synthesis of ornithine transaminase exceeding the basal level [i.e. $0 \cdot 2$ to $0.4 \mu \mathrm{mol} \mathrm{h}^{-1}(\mathrm{mg} \text { protein })^{-1}$ ]. Arginase was also induced 
Table 4. Average specific activities of arginase and ornithine transaminase in diploid yeast strains grown with different nitrogen sources

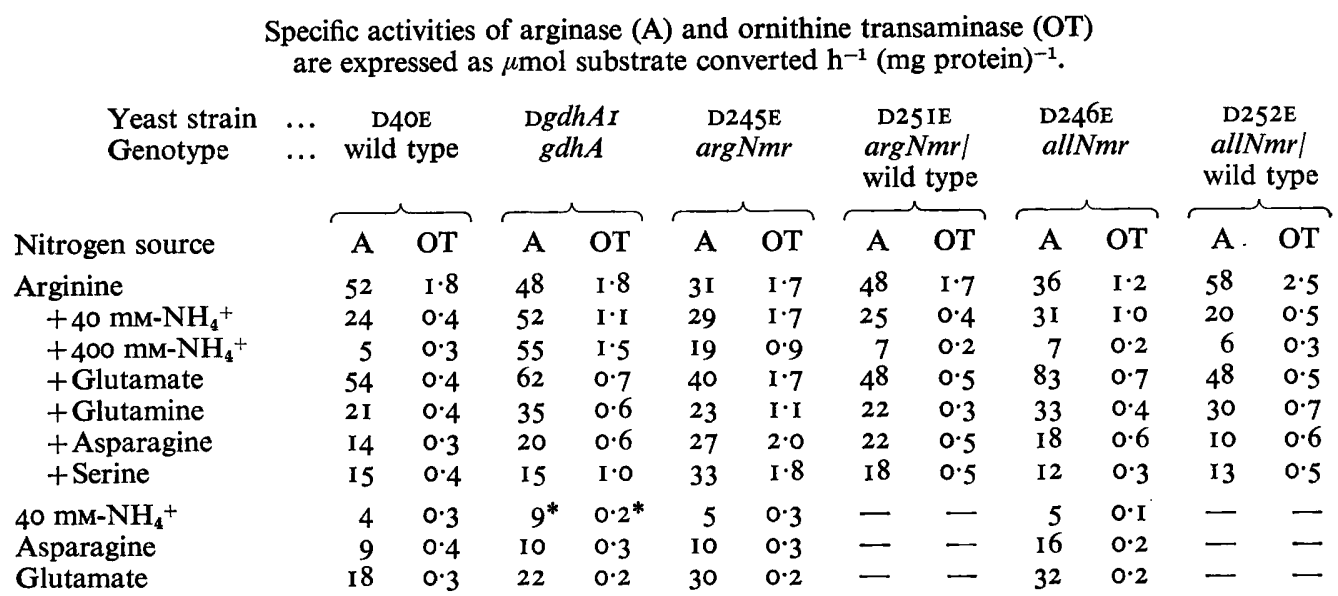

* Specific growth rates, $0.15 \mathrm{~h}^{-1}$; specific growth rates of other cultures, 0.35 to $0.41 \mathrm{I} \mathrm{h}^{-1}$.

in the wild-type $\alpha-\Sigma_{1} 278 \mathrm{~b}$ and related yeast strains during growth on media with glutamate, leucine and some other amino acids (Dubois \& Wiame, 1976), but not in another strain of $S$. cerevisiae (Middelhoven, I970). The enzymes responded differently to nitrogen metabolite repression, ornithine transaminase synthesis being very sensitive and arginase synthesis being repressed completely only by very high $(400 \mathrm{~mm})$ concentrations of ammonium chloride and, to a lesser extent, by $40 \mathrm{~mm}$-ammonium chloride, glutamine, asparagine and serine when added to glucose/arginine medium. In DgdhAr the induction of arginase by arginine was repressed only by asparagine and serine, whereas the induction of ornithine transaminase was also repressed by other nitrogenous compounds. Nitrogen metabolite repression in the $\operatorname{argNmr}$ diploid D245E was relieved to a greater extent, enzyme synthesis being partly inhibited only by $400 \mathrm{mM}$-ammonium chloride and glutamine. The allNmr mutation (D246E) relieved arginase and ornithine transaminase synthesis from repression by $40 \mathrm{~mm}$-ammonium chloride; the repression by other nitrogenous compounds was almost the same as in the wild type. The behaviour of the heterozygous diploids D25IE and D252E indicated that the arg Nmr and allNmr mutations were both recessive to wild type.

The maximum specific arginase activities in strains D245E and D246E, after growth in arginine medium, were lower than that in the wild type. For the $\operatorname{argNmr}$ strain D245E, this was an effect of growth conditions since the arginase activity in cells grown in Kluyver flasks was as high as that in the wild-type strain D4OE. However, the arginase activity of strain D246E (allNmr) was always significantly lower than that of strain D4OE, an effect attributable to the allNmr mutation.

Previously the derepression of arginase and ornithine transaminase during nitrogen starvation of yeast cells, grown in the absence of an inducer, has been a valuable tool in the study of nitrogen metabolite repression (Middelhoven, 1968, 1970; Whitney \& Magasanik, 1973). However, strain $\alpha-\Sigma$ I278b and its derivatives did not show this phenomenon in a reproducible way, and so derepression during nitrogen starvation was not included in this study. 
Table 5. Maximum specific growth rates and substrate constants for ammonium obtained for diploid yeast strains grown in ammonium-limited continuous cultures

$\begin{array}{cc}\text { Yeast strain } & \text { Genotype } \\ \text { D4OE } & \text { wild type } \\ \text { D245E } & \text { argNmr } \\ \text { D246E } & \text { allNmr }\end{array}$

$\begin{array}{cc}\begin{array}{c}\text { Maximum specific } \\ \text { growth rate }\left(\mathrm{h}^{-1}\right)\end{array} & \begin{array}{c}\text { Substrate constant } \\ \text { for ammonium }\left(\mu_{\mathrm{M}}\right)\end{array} \\ 0.4 \mathrm{I} & 5-\mathrm{I} \mathrm{I} \\ 0.40 & 5-6 \\ 0.42 & 9\end{array}$

\section{Genetic characteristics}

The recessivity of the allNmr and argNmr mutations to wild type and the I : I distribution of wild and Nmr phenotypes among segregants obtained by sporulation of heterozygous $\mathrm{Nmr} /$ wild type diploids have been described above. Sporulation of heterozygous diploids allNmr/argNmr, allNmr/allantoinase-less, allNmr/gdhA, $\operatorname{argNmr} / g d h A, \operatorname{argNmr} / \operatorname{arginase-}$ less and $\operatorname{argNm} /$ ornithine transaminase-less resulted in a random segregation of the different markers (results not presented). Segregant strains bearing two markers could be readily isolated. These results imply that the allNmr, argNmr and $g d h A$ mutations belong to different linkage groups and that the former two loci represent true regulatory genes without operator characteristics. If the $\mathrm{Nmr}$ loci were to have an operator function, linkage to structural genes would have been observed.

\section{Ammonium assimilation}

Roon et al. (1975a) demonstrated that, in their yeast strain, methylammonium resistance is caused by defective transport of methylammonium and ammonium into the cell. Arst \& Page (1973) suggested that in $A$. nidulans methylammonium resistance is also accompanied by defective ammonium transport. Although the allNmr and arg Nmr mutants showed the same growth rates on ammonium medium as the wild-type strain, the maximum specific growth rates $\left(\mu_{\mathrm{m}}\right)$ and the substrate constants for the ammonium ion were determined in ammonium-limited chemostat cultures (Middelhoven et al., 1976a). No significant differences between $\mathrm{Nmr}$ diploids and the wild type were observed (Table 5). Thus the $\mathrm{Nmr}$ loci represent true regulatory genes having no effect on ammonium transport.

\section{Glutamate dehydrogenases}

Previously described yeast mutants with relieved nitrogen metabolite repression of arginase or allantoinase are either devoid of anabolic NADP-specific glutamate dehydrogenase ( $g d h A$; Dubois et al., I973) or have derepressed levels of catabolic NAD-specific glutamate dehydrogenase ( $g d h C R$; Dubois \& Grenson, 1974). Identity of the Nmr mutations and the $g d h A$ mutation has been ruled out on the basis of genetic experiments, methylammonium resistance and the rate of ammonium assimilation, but identity of one of the $N m r$ mutants and the $g d h C R$ mutation (not included in this study) is possible. To investigate this possibility, both glutamate dehydrogenases were determined in yeast grown with ammonium, with ammonium/glutamate and with glutamate. In all strains NADspecific glutamate dehydrogenase was induced only in glutamate medium without ammonium (Table 6). The specific activity of this enzyme in D246E (allNmr) grown in glutamate medium was somewhat lower than that in the other strains. The nitrogen source had little influence on NADP-specific glutamate dehydrogenase synthesis. Its activity in both $N m r$ diploids was somewhat higher than in the wild-strain $\alpha-\Sigma_{1278} b$. These results indicate that the $N m r$ mutations are not identical with either the $g d h A$ or the $g d h C R$ mutations. 
Table 6. Average specific activities of NAD-specific and NADP-specific glutamate dehydrogenases in diploid yeast strains grown with different nitrogen sources

Specific activities are expressed as $\mu \mathrm{mol}$ substrate converted $\mathrm{h}^{-1}$ (mg protein) ${ }^{-1}$.

\begin{tabular}{|c|c|c|c|c|c|c|c|c|}
\hline \multirow{2}{*}{$\begin{array}{l}\text { Yeast strain } \\
\text { Genotype }\end{array}$} & \multicolumn{2}{|c|}{$\begin{array}{l}\alpha-\Sigma_{\operatorname{I} 278 b} \\
\text { wild type }\end{array}$} & \multicolumn{2}{|c|}{$\begin{array}{c}\text { D245E } \\
\operatorname{argNmr}\end{array}$} & \multicolumn{2}{|c|}{$\begin{array}{c}\mathrm{D} 246 \mathrm{E} \\
\text { all } N m r\end{array}$} & \multicolumn{2}{|c|}{$\begin{array}{c}\mathrm{D} g d h A I \\
g d h A\end{array}$} \\
\hline & NAD & NADP & NAD & NADP & NAD & NADP & NAD & NADP \\
\hline $\mathrm{NH}_{4}^{+}$ & 0.53 & $20 \cdot 0$ & $I \cdot 08$ & $60 \cdot 0$ & 0.74 & $79 \cdot 0$ & $0.10^{*}$ & $0.2^{*}$ \\
\hline $\mathrm{NH}_{4}^{+}+$Glutamate & 0.53 & 16.5 & 0.45 & $53 \cdot 0$ & 0.52 & $47 \cdot 0$ & 0.53 & 0.2 \\
\hline Glutamate & $4 \cdot 8$ & $35 \cdot 1$ & $7 \cdot 6$ & $50 \cdot 0$ & $2 \cdot 0$ & $53 \cdot 0$ & $4 \cdot 7$ & $\mathrm{I} \cdot 2$ \\
\hline
\end{tabular}

* Specific growth rates, about $0.15 \mathrm{~h}^{-1}$; specific growth rates of other cultures, 0.35 to $0.4 \mathrm{I} \mathrm{h}^{-1}$.

\section{Other methylammonium-resistant mutants}

Besides mutants of the allNmr and $\operatorname{argNm}$ types, a third genetic class of methylammoniumresistant mutants was frequently isolated on plates with various nitrogen sources (e.g. DLallantoin, L-arginine, DL-2-aminobutyrate). In these mutants allantoinase synthesis was repressed by ammonium/glutamate, asparagine and glutamine but not by ammonium chloride. The mutation was semi-dominant with respect to the latter property. Heterozygous diploids with the wild type showed allantoinase activities halfway between those in the parent strains when grown on ammonium or on allantoin/ammonium medium. However, these mutants did not possess a true regulatory mutation, since the substrate constant for ammonium in them was 50 to $100 \mu \mathrm{M}$. The latter was considerably higher than the values for wild-type and $\mathrm{Nmr}$ mutants (Table 5). Although growth rates in ammonium medium were normal, this class of allantoin/methylammonium-resistant mutants was probably defective in ammonium transport. It is therefore difficult to understand why repression by ammonium was relieved only in allantoinase synthesis and not in arginine catabolism.

\section{DISCUSSION}

This paper deals with two novel mutations, allNmr and $\operatorname{argNmr}$, that cause impaired nitrogen metabolite repression of enzymes involved in allantoin and arginine catabolism. Yeast strains carrying these mutations were selected on the basis of their resistance to methylammonium chloride. The cause of the toxicity of this substance and the mechanism of its action are unknown. In the wild-type strain D4OE sensitivity to methylammonium varied considerably with the nitrogen source (Table 2). Methylammonium resistance has been attributed to defective ammonium transport (Arst \& MacDonald, I973; Roon et al., I975a). Although some mutants with defective ammonium transport were found among allantoin/methylammonium-resistant mutants, isolated in the present study, ammonium transport by allNmr and arg Nmr strains was as efficient as in the wild type (Table 5).

The obligatory role of allophanate as an inducer of allantoinase synthesis (Cooper \& Lawther, 1973) was not apparent in our experiments, as enzyme activities in cultures grown in alanine or 2-aminobutyrate medium were higher than those in cultures grown with the allophanate-generating nitrogen sources allantoin, urea and arginine. Nitrogen metabolite repression of allantoinase synthesis was little affected by the gdhA (cf. van de Poll, 1973) and argNmr mutations but was considerably relieved by the allNmr mutation.

Nitrogen metabolite repression of arginase synthesis in the wild-type strain D4OE was less pronounced than in a yeast strain previously used (Middelhoven, 1970). Moreover, 
the depression during nitrogen starvation of arginase and ornithine transaminase shown by yeast strain $\alpha-\Sigma_{1} 278 b$ and its derivatives was less significant and reproducible than in yeast strains used in previous studies (Middelhoven, 1968, 1970; Whitney \& Magasanik, 1973). This suggests that considerable differences in regulation of enzyme synthesis may exist between different yeast strains. Nitrogen metabolite repression of arginase was greatly relieved by the $g d h A$ mutation (cf. Dubois et al., 1973, 1974) but was still exerted by serine and asparagine. The repression of ornithine transaminase by several nitrogenous substances, except ammonium, was hardly affected by the $g d h A$ mutation (cf. Dubois et al., 1973). Nitrogen metabolite repression of both arginase and ornithine transaminase synthesis was considerably relieved by the argNmr mutation, although the sensitivity to glutamine and to very high ammonium concentrations was partly retained. The all Nmr mutation had only a small effect on nitrogen metabolite repression of arginase and ornithine transaminase: it relieved enzyme synthesis from repression by $40 \mathrm{mM}$-ammonium chloride but barely from that by other nitrogen sources.

Both the allNmr and the argNmr mutations are recessive to wild type with respect to all phenotypic properties tested. The genes are not linked to each other, nor to the $g d h A$ locus, nor to the structural genes of allantoinase, arginase and ornithine transaminase. Hence they represent regulatory mutations without operator function, different from known mutations with impaired nitrogen metabolite repression ( $g d h A$ and $g d h C R$ ). The $g d h C R$ mutation is characterized by derepression synthesis of catabolic NAD-specific glutamate dehydrogenase (Dubois \& Grenson, I974), a property not shown by the allNmr and $\operatorname{argNmr}$ mutants (Table 6).

The $\operatorname{argNmr}$ locus appears to function in the nitrogen metabolite repression of enzymes involved in arginine catabolism. It is probably pathway-specific as pleiotropic effects were not observed, except for an increased resistance to methylammonium in leucine and glutamate media and a slight derepression of NADP-specific glutamate dehydrogenase (Table 6). The allNmr locus, however, appears to have a more general function. Besides a considerable relief of allantoinase synthesis from nitrogen metabolite repression and the coincident methylammonium resistance in allantoin medium, several pleiotropic effects of the all Nmr mutation were observed. The synthesis of arginase and ornithine transaminase in allNmr mutants was insensitive to repression by $40 \mathrm{mM}$-ammonium chloride (Table 4 ); maximum specific activities of arginase and NAD-specific glutamate dehydrogenase (Table 6) were lower than in the wild type, whereas NADP-specific glutamate dehydrogenase was slightly derepressed as in arg Nmr mutants. Moreover, the allNmr mutation led to methylammonium resistance in media with several amino acids as the sole nitrogen source (Table 2).

Our results support the conclusions of others (van de Poll, r973; Bossinger \& Cooper, I975; Roon et al., I975 $b$; Hynes, 1974) questioning the role of the $g d h A$ gene in nitrogen metabolite repression (Dubois et al., 1973, 1974). Neither allantoinase nor arginase synthesis is completely insensitive to repression by nitrogenous compounds in $g d h A$ mutants.

The data presented in this paper shed some light on the mechanism of nitrogen metabolite repression in yeast. This regulatory device appears to be achieved by the cooperation of several genes, some of which (argNmr) have a pathway-specific function and some ( $g d h C R$, allNmr) a more general function. The role of the gdhA mutation in nitrogen metabolite repression is questionable. The involvement in nitrogen metabolite repression of allantoin and arginine catabolism of other genes is to be expected as neither the all Nmr nor $\operatorname{argNmr}$ mutations result in a complete relief of nitrogen metabolite repression. The role of other genes in this regulatory system is being investigated. 
Thanks are due to Mrs M. C. Hoogkamer-te Niet, Mrs A. Legger-Huysman, Mr P. R. Croes, Mr H. P. van Egmond and Mr A. W. H. M. Meijs for their skilful technical assistance.

\section{REFERENCES}

ARST, H. N., JR \& Cove, D. J. (1969). Methylammonium resistance in Aspergillus nidulans. Journal of Bacteriology 98, 1284-I294.

ARST, H. N., JR \& MACDONALD, D. W. (1973). A mutant of Aspergillus nidulans lacking NADP-linked glutamate dehydrogenase. Molecular and General Genetics 122, 26I-265.

ARsT, H. N., JR \& PAGE, M. M. (1973). Mutants of Aspergillus nidulans altered in the transport of methylammonium and ammonium. Molecular and General Genetics 121, 239-245.

BÉCHET, J., Grenson, M. \& WIAME, J. M. (1970). Mutations affecting the repressibility of arginine biosynthetic enzymes in Saccharomyces cerevisiae. European Journal of Biochemistry 12, 3I-39.

BOssinger, J. \& COOPER, T. G. (1975). Possible failure of NADP-glutamate dehydrogenase to participate directly in nitrogen repression of the allantoin degradative enzymes in Saccharomyces cerevisiae. Biochemical and Biophysical Research Communications 66, 889-892.

BOSSINGER, J., LAWTHER, R. P. \& COOPER, T. G. (1974). Nitrogen repression of the allantoin degradative enzymes in Saccharomyces cerevisiae. Journal of Bacteriology 118, 82 I-829.

COOPER, T. G. \& LAWTHER, R. P. (1973). Induction of the allantoin degradative enzymes in Saccharomyces cerevisiae by the last intermediate of the pathway. Proceedings of the National Academy of Sciences of the United States of America 70, 2340-2344.

DiCarlo, F. J., Schultz, A. S. \& McManus, D. K. (195I). The assimilation of nucleic acid derivatives and related compounds by yeast. Journal of Biological Chemistry 189, I $5 \mathrm{I}-157$.

DiCarlo, F. J., Schultz, A. S. \& Kent, A. M. (1953). The mechanism of allantoin catabolism by yeast. Archives of Biochemistry and Biophysics 44, 468-474.

DOHERTY, D. (1970). L-Glutamate dehydrogenases (yeast). Methods in Enzymology 17, 850-856.

Dubors, E. \& GRENSON, M. (1974). Absence of involvement of glutamine synthetase and of NAD-linked glutamate dehydrogenase in the nitrogen catabolite repression of arginase and other enzymes in Saccharomyces cerevisiae. Biochemical and Biophysical Research Communications 60, 150-157.

DuBoIs, E. \& WIAME, J. M. (1976). Non specific induction of arginase in Saccharomyces cerevisiae. Biochimie 58, 207-21I.

Dubois, E., Grenson, M. \& WiAME, J. M. (I973). Release of the ammonia effect on three catabolic enzymes by NADP-specific glutamate dehydrogenase-less mutations in Saccharomyces cerevisiae. Biochemical and Biophysical Research Communications 50, 967-972.

DuBois, E., Grenson, M. \& WIAME, J. M. (1974). The participation of the anabolic glutamate dehydrogenase in the nitrogen catabolite repression of arginase in Saccharomyces cerevisiae. European Journal of Biochemistry 48, 603-616.

Fink, G. R. (1970). The biochemical genetics of yeast. Methods in Enzymology 17A, 59-78.

GITS, J. J. \& GRENSON, M. (I967). Multiplicity of the amino acid permeases in Saccharomyces cerevisiae. III. Evidence for a specific methionine-transporting system. Biochimica et biophysica acta 135, 507-516.

Grenson, M. \& Hov, C. (1972). Ammonia inhibition of the general amino acid permease and its suppression in NADPH-specific glutamate dehydrogenase-less mutants in Saccharomyces cerevisiae. Biochemical and Biophysical Research Communications 48, 749-756.

HaGan, J. J. \& DallaM, R. D. (1968). Measurement of arginase activity. Analytical Biochemistry 22, $518-$ 524.

HoRN, P. \& WILKIE, D. (1966). Use of Magdala red for the detection of auxotrophic yeast mutants of Saccharomyces cerevisiae. Journal of Bacteriology 91 , 1388.

HYNES, M. J. (1974). Effects of ammonium, L-glutamate, and L-glutamine on nitrogen catabolism in Aspergillus nidulans. Journal of Bacteriology $\mathbf{1 2 0}$, I $116-1123$.

KINGHORN, J. R. \& PATEMAN, J. A. (I973). NAD and NADP L-glutamate dehydrogenase activity and ammonium regulation in Aspergillus nidulans. Journal of General Microbiology 78, 39-46.

LARUe, T. A. \& SPEnCER, J. F. T. (1968). The utilization of purines and pyrimidines by yeasts. Canadian Journal of Microbiology 14, 78-86.

MidDELHOVEN, W. J. (1964). The pathway of arginine breakdown in Saccharomyces cerevisiae. Biochimica et biophysica acta $93,650-652$.

MidDelhoven, W. J. (1968). The derepression of arginase and of ornithine transaminase in nitrogen-starved baker's yeast. Biochimica et biophysica acta 156, 440-443.

MIDDELHOVEN, W. J. (1969a). The ferrous ion as the cofactor of arginase in vivo. I. Properties of yeast arginase metallo-complexes of known composition and of native arginases. Biochimica et biophysica acta I9I, I IO-I21.

MidDELHOVEN, W. J. (1969b). The effect of myo-inositol on the synthesis of arginase and ornithine transaminase in baker's yeast. Biochimica et biophysica acta 192, 243-251. 
Middelhoven, W. J. (1969c). Enzyme repression in the arginine pathway of Saccharomyces cerevisiae. Antonie van Leeuwenhoek 35, 2 I5-226.

Middelhoven, W. J. (1970). Induction and repression of arginine and ornithine transaminase in baker's yeast. Antonie van Leeuwenhoek 36, I-19.

Middelhoven, W. J., DE WAaRd, M. A. \& Mulder, E. G. (1969). The ferrous ion as the cofactor of arginase in vivo. II. Experiments on the replacement of ferrous ions in native yeast arginase by other cations in vivo. Biochimica et biophysica acta r91, I22-129.

Middelhoven, W. J., Anderegg, M. J. P. T., Meys, A. W. H. M. \& van Egmond, H. P. (I976a). The substrate constant for the ammonium ion of growing Saccharomyces cerevisiae. Antonie van Leeuwenhoek 42, 293-297.

Middelhoven, W. J., Broekruizen, B. \& van Eijk, J. (1976 b). The detection of yeast mutants unable to utilize nitrogenous substances as the sole nitrogen source with the dye phloxine B. Journal of Bacteriology I28, 85I-852.

Parker, J. H. \& Mattoon, J. R. (I969). Mutants of yeast with altered oxidative energy metabolism: selection and genetic characterization. Journal of Bacteriology 100, 647-657.

VAN DE POLL, K. W. (1970). Repression and derepression of allantoinase synthesis in protoplasts of Saccharo-

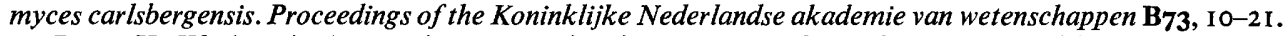

VAN DE Poll, K. W. (1973). Ammonium repression in a mutant of Saccharomyces carlsbergensis lacking NADP dependent glutamate dehydrogenase activity. FEBS Letters 32, 265-266.

RoON, R. J. \& LevenberG, B. ( 1970 ). $\mathrm{CO}_{2}$ fixation and the involvement of allophanate in the biotin-enzymecatalyzed cleavage of urea. Journal of Biological Chemistry 245, 4593-4595.

Roon, R. J., Even, H. L., DunloP, P. \& LARIMORE, F. L. (1975a). Methylamine and ammonia transport in Saccharomyces cerevisiae. Journal of Bacteriology 122, 502-509.

Roon, R. J., LARImore, F. \& LeVY, S. J. (I975b). Inhibition of amino acid transport by ammonium ion in Saccharomyces cerevisiae. Journal of Bacteriology 124, 325-331.

Vogels, G. D. \& VAN DER DRIFT, C. (1970). Differential analysis of glyoxylate derivatives. Analytical Biochemistry 33, I43-157.

Whitney, P. A. \& CoOper, T. G. (1972). Urea carboxylase and allophanate hydrolase: two components of a multienzyme complex in Saccharomyces cerevisiae. Biochemical and Biophysical Research Communications 49, 45-5I.

Whitney, P. A. \& Magasanik, B. (I973). The induction of arginase in Saccharomyces cerevisiae. Journal of Biological Chemistry 248, 6197-6202.

Whitney, P. A., Cooper, T. G. \& MAGasanik, B. (I973). The induction of urea carboxylase and allophanate hydrolase in Saccharomyces cerevisiae. Journal of Biological Chemistry 248, 6203-6209. 